\title{
Self-Reported Sleep Quality With Mandibular Advancement Device or Continuous Positive Airway Pressure: A Randomized Clinical Trial on Patients With Mild and Moderate Obstructive Sleep Apnea
}

\author{
Lars Martin Berg, DDS ${ }^{1}$; Torun Karina S. Ankjell, MD²; Tordis Agnete Trovik, DDS, MPH, PhD³; Anders Sjögren, DDS, PhD; \\ Oddveig G. Rikardsen, MD, PhD²; Ketil Moen, DDS, PhD ${ }^{4}$; Yi-Qian Sun, PhD ${ }^{5}$; Vegard Bugten, MD, PhD ${ }^{6}$
}

\begin{abstract}
${ }^{1}$ Department of Clinical Dentistry, Faculty of Health Sciences, UiT The Arctic University of Norway, Troms $\varnothing$, Norway; ${ }^{2}$ ENT Department, University Hospital in Northern Norway, Troms $\varnothing$, Norway, and Department of Clinical Medicine, Faculty of Health Sciences, UiT The Arctic University of Norway, Troms $\emptyset$, Norway; ${ }^{3}$ Department of Community Medicine, Faculty of Health Sciences, UiT The Arctic University of Norway, Troms $\varnothing$, Norway; ${ }^{4}$ ENT Department, Section for Oral and Maxillofacial Surgery, Arendal Hospital, Arendal, Norway; ${ }^{5}$ Center for Oral Health Services and Research, Mid-Norway (TkMidt), Trondheim, Norway, and Department of Clinical and Molecular Medicine, Faculty of Medicine and Health Sciences, NTNU Norwegian University of Science and Technology, Trondheim, Norway; ${ }^{6}$ Department of Otorhinolaryngology, Head and Neck Surgery, St. Olav's University Hospital, Trondheim, Norway, and Department of Neuromedicine and Movement Science, Faculty of Medicine and Health Sciences, NTNU Norwegian University of Science and Technology, Trondheim, Norway
\end{abstract}

\begin{abstract}
Study Objectives: To compare self-reported sleep quality, treatment compliance, and respiratory event index (REI) after 4 months of treatment with mandibular advancement device (MAD) or continuous positive airway pressure (CPAP) in mild and moderate obstructive sleep apnea (OSA).
\end{abstract}

Materials and Methods: A total of 104 patients with mild or moderate OSA were randomly allocated to MAD or CPAP treatment and followed for 4 months. Data were collected through type 3 polygraphic sleep recordings, CPAP recordings, medical examination, and the Pittsburgh Sleep Quality Index (PSQI). Chi-square test, t-test, and Mann-Whitney U test were used to analyze compliance, PSQI global score and REI, respectively. Reliable change index (RCI) was used to evaluate change in PSQI global score.

Results: Six patients were lost to follow-up. More patients were compliant with MAD treatment (79.5\%) than CPAP treatment (38.9\%) at follow-up $(\mathrm{P}<0.001)$. Both groups had improved PSQI global scores: MAD $(8.0 \pm 3.1$ to $5.7 \pm 2.5, \mathrm{P}<0.001)$ and $\mathrm{CPAP}(7.7 \pm 3.5$ to $6.7 \pm 3.4, \mathrm{P}=0.01)$. More patients had improved PSQI global score according to the RCI in the MAD group (38.6\%) than in the CPAP group $(16.7 \%)(\mathrm{P}=0.01)$. Both treatments reduced REI $(\mathrm{P}<0.001)$, but CPAP $(\mathrm{REI}=1.1)$ more so than MAD $(\mathrm{REI}=7.9)(\mathrm{P}<0.001)$.

Conclusions: Both MAD and CPAP treatment improve self-reported sleep quality in patients with mild and moderate OSA. More patients comply with MAD treatment, which improves sleep quality in more patients than does CPAP, despite REI being lower in the CPAP group. With respect to sleep quality, MAD treatment should be considered a better treatment option than CPAP in mild and moderate OSA.

Keywords: adult; continuous positive airway pressure; mandibular advancement; obstructive sleep apnea; occlusal splints; patient compliance; respiratory disorders; self-report; sleep hygiene

Citation: Berg LM, Ankjell TKS, Trovik TA. Self-Reported Sleep Quality With Mandibular Advancement Device or Continuous Positive Airway Pressure: A Randomized Clinical Trial on Patients With Mild and Moderate Obstructive Sleep Apnea. J Dent Sleep Med. 2020;7(2)

\section{INTRODUCTION}

Obstructive sleep apnea (OSA) is characterized by repeated collapse of the soft tissues in the upper airway and leads to sleep fragmentation and reduced sleep quality. ${ }^{1-3}$ The severity of OSA is measured by the apnea-hypopnea index (AHI) and is graded as mild (AHI 5-14.9 events/h), moderate (AHI 15-29.9 events/h) and severe (AHI >30 events/h). ${ }^{4}$ When OSA is diagnosed through unattended sleep apnea testing the AHI is substituted by the respiratory event index (REI). ${ }^{5}$ Continuous positive airway pressure (CPAP) reduces respiratory events by eliminating the negative respiratory pressure that collapses the upper airways. ${ }^{6}$ Despite challenges regarding patient adherence, CPAP is currently regarded as the gold standard for treatment of all patients with $\mathrm{OSA}^{7}{ }^{7}$ whereas exercise training and weight loss are recommended as adjunct treatments for all patients with OSA who are overweight. ${ }^{8,9}$ In addition, surgical interventions are indicated in selected groups of patients with OSA. ${ }^{10-12}$ For patients with primary snoring or mild OSA or those who are unable or unwilling to use CPAP, a mandibular advancement device (MAD) is regarded as an adequate alternative. ${ }^{7,13,14}$ MADs relocate and fixate the mandible in a protruded position when used, thus increasing the retropalatal and retroglossal volume and thereby reducing the collapsibility of the upper airways. ${ }^{15,16}$ Although CPAP treatment is known to be superior to MAD treatment in regard to respiratory event reduction, ${ }^{7,10,17}$ several studies have shown patients adhering better to MAD and preferring MAD over CPAP when given the choice. ${ }^{18-21}$ Both CPAP and MAD treatments are associated with only mild and transient adverse effects such as 
pressure in the face, mild pain in teeth and jaw, or changes in salivation, ${ }^{10,16,19,22}$ suggesting that factors other than adverse effects from CPAP and MAD have an effect on the patient's preferences and motivation in OSA treatment. ${ }^{15,20}$ The patient's perceived sleep quality while using CPAP or MAD might be one such factor, but self-reported sleep quality during OSA treatment is not extensively studied. ${ }^{17,23}$ Contrary to polysomnographic analysis of sleep quality, which shows sleep efficacy and changes in sleep stages during OSA treatment, ${ }^{24,25}$ self-reported sleep quality shows how the patient experiences the effect of OSA treatment on sleep quality. It is currently not clear which OSA treatment is better at improving self-reported sleep quality, or how much improvement could be expected in patients with mild and moderate OSA. ${ }^{2}$ To our knowledge, no randomized controlled trial has previously measured and compared the effect of CPAP and MAD treatment on self-reported sleep quality in patients with mild and moderate OSA.

\section{Aim}

In this study we aimed to compare self-reported sleep quality after initial phase (4 months) of MAD or CPAP treatment in mild and moderate OSA. Secondary aims were to compare treatment compliance and change in REI from baseline to follow-up.

\section{MATERIALS AND METHODS}

\section{Study Design}

This study was a two-centered parallel arm randomized controlled clinical trial, with 50/50 allocation ratio. Blinding of the patients and clinical personnel was not feasible because of the nature of the OSA treatment.

\section{Ethical Approval}

The trial was approved by the Norwegian Regional Committee for Medical and Health Research Ethics, REC Central (registration \#2014/956) and was registered in ClinicalTrials.gov (registration \#NCT02953028).

\section{Calibration}

Two researchers (LMB and TKSA) calibrated all personnel involved in the patient treatment and data collection according to the trial protocol. The protocol checklists complied with the later updated American Academy of Sleep Medicine practice guidelines for diagnostic testing for OSA. ${ }^{26}$

\section{Recruitment and Randomization}

All patients in the trial were referred from primary health care to the ear-nose-throat departments at the University hospital of Northern Norway (UNN) in Tromsø, St. Olavs University Hospital (St. Olavs), and Aleris Hospital in Trondheim, Norway. All patients were screened for OSA by ambulatory type 3 sleep recording devices (Embletta ${ }^{\circledR}$ or Nox T3 ${ }^{\mathrm{TM}}$, ResMed Norway AS) at home or at a hotel. Respiratory events were defined as $>90 \%$ reduction in respiratory flow or $>50 \%$ reduction in respiratory flow combined with $\geq 3 \%$ oxygen desaturation from baseline respectively. The sleep recordings were manually analyzed by sleep technicians at the three hospitals before otorhinolaryngologists at UNN and St. Olavs Hospitals performed a medical examination of the patients. All patients meeting the inclusion criteria were invited to participate in the study by the otorhinolaryngologist.

Inclusion criteria were age 20 to 75 years, REI between 10.0 and 29.9, and ability to protrude the mandible at least $5 \mathrm{~mm}$. Exclusion criteria were pregnancy, drug abuse, daily use of sedative medication, preexisting severe psychiatric disorders, or somatic health issues such as temporomandibular dysfunction that prevented the use of CPAP or MAD.

After providing informed written consent to participate, the patients were randomized to treatment with either CPAP or MAD. The patients drew lots from a masked envelope for random allocation. Blockrandomization with 30 lots per block at each of the two study sites was used to prevent skewed distribution between CPAP and MAD groups across seasons and study sites.

\section{Treatment Protocol and Questionnaires}

The treatment protocol was based on the recommendations from the Standards of Practice Committee and the Board of Directors of the American Academy of Sleep Medicine. ${ }^{27}$ Patients allocated to the CPAP group met a sleep technician on 2 consecutive days for adaptation and adjustment of the CPAP-machine (Resmed ${ }^{\circledR}$, San Diego, California, USA). A facemask or nose mask was used depending on the patient's needs and preference. The patients had the opportunity to see the sleep technicians for adjustments of the CPAP machine when necessary.

Patients allocated to the MAD group met a dentist and dental hygienist or dental nurse for impression of the dentition, bite registration using the George gauge ${ }^{\mathrm{TM}}$ (Scheu-dental GmbH, Iserlohn, Germany) and ordering the MAD (Respire Medical, New York, New York, USA and SomnoDent ${ }^{\circledR}$, Sydney, New South Wales, Australia). At the second visit to the dental team the MAD was set between $60 \%$ and $65 \%$ of maximum protrusion of the mandible. Necessary changes to the MAD based on patient feedback were made after 2 to 3 weeks. The patients had the opportunity to see the dental team for further 
adjustments of the MAD when necessary.

At the medical examination prior to treatment, all patients completed the Pittsburgh Sleep Quality Index (PSQI) a 19-item validated questionnaire measuring selfperceived sleep quality during the previous month. PSQI assess 7 aspects of sleep quality: subjective sleep quality, sleep latency, sleep duration, habitual sleep efficiency, sleep disturbances, use of sleeping medicine, and daytime dysfunction, which are transformed into a global score. The PSQI global score has a possible range of 0-21 points, with $\leq 5$ points representing good sleep quality. ${ }^{28}$

To evaluate whether the change in PSQI for each individual patient was clinically and statistically significant, the reliable change index (RCI) as described by Jacobson and Truax ${ }^{29}$ was used. The RCI was calculated by using the standard deviation from pretreatment PSQI global score and a test-retest reliability at $0.85 .{ }^{28} \mathrm{An}$ RCI value $<-1.96$ indicate that the patient's reduction in PSQI global score is statistically significant on a $5 \%$ level, and thus not likely to occur due to expected test-retest variations. The patients also completed a 12-item questionnaire assessing general health, socioeconomic status, and smoking and alcohol habits.

At follow-up after approximately 4 months, all patients completed the PSQI questionnaire and a questionnaire covering self-reported compliance to both the CPAP and MAD group. Recordings of usage and REI were downloaded from the CPAP machine, whereas patients using MAD recorded REI through a new polygraphic sleep recording while using the MAD. Sleep technicians at UNN and St. Olavs hospitals analyzed both CPAP recordings and the new polygraphic sleep recordings. Patients were regarded as compliant with treatment if they reported using the CPAP or MAD more than 4 hours during more than $50 \%$ of nights.

\section{Statistical Analysis}

Both intention-to-treat (ITT) analyses (all included patients) and per-protocol (PP) analyses (patients compliant with treatment) are presented for the primary aim and compliance. ${ }^{30}$ REI was analyzed according to ITT only. At follow-up, the MAD and CPAP groups were compared using the $t$-test (PSQI), Mann-Whitney $U$ test (REI), and Pearson chi-square test (compliance with treatment). Change within treatment groups in PSQI global score and REI from baseline to follow-up was analyzed using paired-samples $t$-test and Wilcoxon signed-rank test, respectively. Missing entries in PSQI were replaced by multiple imputations as recommended by CONSORT $2010 .^{31,32}$ The number of patients with $\mathrm{RCI}<-1.96$ in each treatment group was compared using Pearson chi-square test. All statistical analyses were done using SPSS 25 statistical software package (IBM Corp, Armonk, New York, USA) and a two-sided value of $P<0.05$ was considered statistically significant.
Sample Size

Based on an expected 15\% difference in PSQI global score between treatment groups at follow-up and a common standard deviation within groups at $25 \%$, a sample size of 45 patients in each treatment group was needed to detect differences in a $t$-test between the groups at a 5\% significance level (two-tailed analysis) and reaching $80 \%$ power. Assuming a $10 \%$ dropout rate, a minimum of 99 patients was needed for the trial.

\section{RESULTS}

\section{Participant Flow, Dropouts, and Treatment Compliance}

Patients were recruited to the study between October 2014 and February 2018. A total of 104 patients satisfied the inclusion criteria and signed a written consent to participate in the trial. Forty-nine patients were allocated to MAD treatment and 55 were allocated to CPAP treatment. The flow of patients in the study is illustrated in Figure 1. Thirty-eight patients with a MAD and 45 patients using CPAP had no missing data throughout the trial. After replacing missing PSQI entries through imputations, 44 patients using a MAD and 54 patients using CPAP were included in the PSQI analyses at 4 months. Median time from treatment start to follow-up was 4 months, range 2 to 8 months. Follow-up was ended in January 2019.

The 11 patients ceasing treatment before follow-up were demographically similar to the remaining study population at baseline and were evenly distributed between mild and moderate OSA. Information on compliance was available for 98 patients. The chi-square test showed more patients being compliant with MAD treatment $(79.5 \%)$ than CPAP treatment (38.9\%) at follow-up $(P<0.001)$.

\section{Baseline Data and Outcome Variables}

Demographic patient data at baseline are presented in Table 1 and were uniformly distributed between the treatment groups. The distribution was similar in the ITT and $\mathrm{PP}$ analysis (supplementary Table S 1).

\section{PSQI Global Score}

From baseline to follow-up, mean PSQI global score was reduced in both treatment groups in the ITT analysis: MAD group from $8.0 \pm 3.1$ to $5.7 \pm 2.5, P<0.001$ and CPAP group from $7.7 \pm 3.5$ to $6.7 \pm 3.4, P=0.01$. $P P$ analysis including only patients with treatment compliance also showed reduced PSQI global score in both treatment groups: MAD group from $8.1 \pm 3.1$ to $5.3 \pm 2.5, P<0.001$ and CPAP group from $7.1 \pm 3.5$ to $5.8 \pm 3.3, P=0.02$. The PSQI global score did not differ between the treatment groups at follow-up (Table 2). 
Figure 1. Patient flow chart

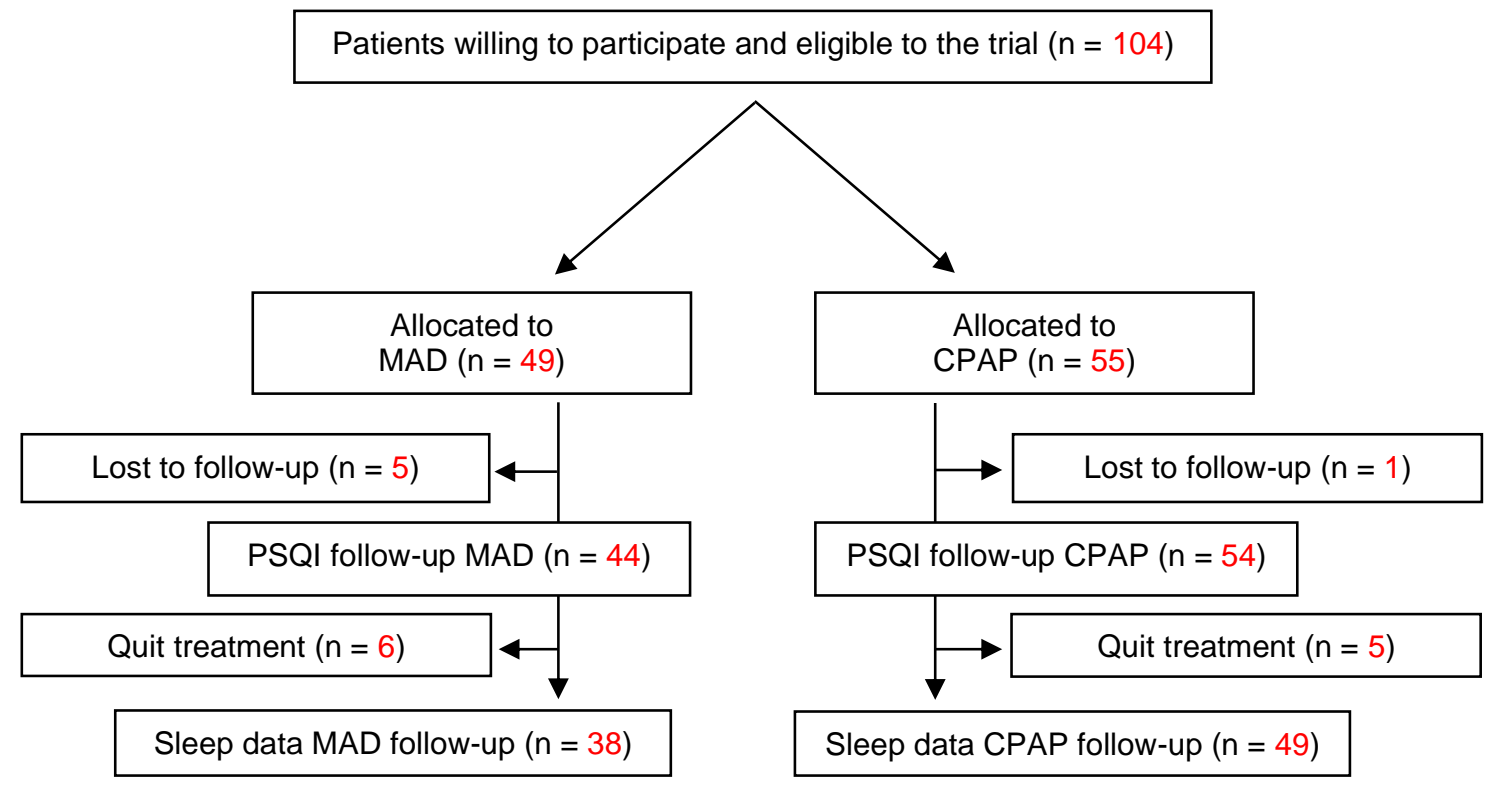

MAD: Mandibular Advancement Device

CPAP: Continuous Positive Airway Pressure

\begin{tabular}{|c|c|c|}
\hline Baseline variables & MAD (n=49) & CPAP (n=55) \\
\hline \multirow{2}{*}{$\begin{array}{l}\text { Age at inclusion } \\
\text { BMI at inclusion }\end{array}$} & $49.6(9.0)$ & $53.3(10.2)$ \\
\hline & $32.4(7.2)$ & $30.8(6.2)$ \\
\hline Female & $20(40.8)$ & $17(30.9)$ \\
\hline Male & 29 (59.2) & $38(69.1)$ \\
\hline \multicolumn{3}{|l|}{ Marital status } \\
\hline Cohabitant & $37(75.5)$ & $44(80.0)$ \\
\hline Living alone & $12(24.5)$ & $11(20.0)$ \\
\hline \multicolumn{3}{|l|}{ OSA severity } \\
\hline Mild & $19(38.8)$ & $13(23.6)$ \\
\hline Moderate & $30(61.2)$ & $42(76.4)$ \\
\hline \multicolumn{3}{|l|}{ Allergy } \\
\hline Yes & 8 (16.3) & $9(16.4)$ \\
\hline No & $51(83.7)$ & $46(83.6)$ \\
\hline \multicolumn{3}{|l|}{ Self-reported health } \\
\hline Good-Excellent & $13(26.5)$ & $16(29.1)$ \\
\hline Poor-Fair & $36(73.5)$ & $39(70.9)$ \\
\hline \multicolumn{3}{|l|}{ Education level } \\
\hline College or university & $27(55.1)$ & $23(41.8)$ \\
\hline Other education & $22(44.9)$ & $32(58.2)$ \\
\hline \multicolumn{3}{|l|}{ Alcohol consumption } \\
\hline $\begin{array}{l}\leq \text { Once per week } \\
>\text { Once per week }\end{array}$ & $40(81.6)$ & $43(78.2)$ \\
\hline \multicolumn{3}{|l|}{ Smoking status } \\
\hline Nonsmoking & $42(85.7)$ & $41(74.5)$ \\
\hline Smoking & $7(14.3)$ & $14(25.5)$ \\
\hline \multicolumn{3}{|c|}{ aMean (standard deviation), all other variables: $\mathrm{n}(\%)$. } \\
\hline \multicolumn{3}{|c|}{$\begin{array}{l}\text { BMI, body mass index }\left(\mathrm{kg} / \mathrm{m}^{2}\right) \text {; CPAP, continuous positive airway } \\
\text { pressure; MAD, mandibular advancement device; OSA, obstructive } \\
\text { sleep apnea. }\left(\mathrm{kg} / \mathrm{m}^{2}\right) \text {. }\end{array}$} \\
\hline
\end{tabular}


Table 2. PSQI and REI at baseline and follow-up.

\begin{tabular}{|c|c|c|c|c|c|}
\hline & \multicolumn{2}{|l|}{ Baseline } & \multicolumn{3}{|c|}{ Follow-up (4 months) } \\
\hline & $\begin{array}{l}\text { MAD } \\
(n=49)\end{array}$ & $\begin{array}{l}\text { CPAP } \\
(n=55)\end{array}$ & $\begin{array}{l}\text { MAD } \\
(n=44)\end{array}$ & $\begin{array}{l}\text { CPAP } \\
(n=54)\end{array}$ & $P$ \\
\hline PSQI ITT & $\begin{array}{l}8.0 \\
(3.1)\end{array}$ & $\begin{array}{l}7.7 \\
(3.5)\end{array}$ & $\begin{array}{l}5.7^{\mathrm{a}} \\
(2.5)\end{array}$ & $\begin{array}{l}6.7^{\mathrm{a}} \\
(3.4)\end{array}$ & .11 \\
\hline PSQI PP & $\begin{array}{l}8.1 \\
(3.1)\end{array}$ & $\begin{array}{l}7.1 \\
(3.5)\end{array}$ & $\begin{array}{l}5.3^{a} \\
(2.5)\end{array}$ & $\begin{array}{l}5.8^{\mathrm{a}} \\
(3.3)\end{array}$ & .55 \\
\hline$R E I^{c}$ & $\begin{array}{l}16.3 \\
(12.4-23.0)\end{array}$ & $\begin{array}{l}18.1 \\
(15.3-24.6)\end{array}$ & $\begin{array}{l}7.9^{a} \\
(6.0-13.7)\end{array}$ & $\begin{array}{l}1.1^{\mathrm{a}} \\
(0.6-1.6)\end{array}$ & $<.001$ \\
\hline
\end{tabular}

$P$ indicates $t$-test/Mann-Whitney $U$ test between MAD and CPAP group at follow-up. Mean (standard deviation). median (interquartile range).

ITT, intention to treat; PP, per protocol; PSQI, Pittsburgh Sleep Quality Index (global score); REI, respiratory event index (events/h).

aStatistically significant change from baseline within treatment group $(P<0.05)$.

bMAD $n=35$ at follow-up, CPAP $n=21$ at follow-up.

cMAD $n=38$ at follow-up, CPAP $n=49$ at follow-up.

\section{Reliable Change in PSQI Global Score}

According to the RCI, significantly more patients in the MAD group than in the CPAP group reported improvement in sleep quality, that is, having RCI $<-1.96$ for the change in PSQI global score from baseline to follow-up. This was found in both the ITT and PP analyses (Table 3).

Table 3. Number of patients with significantly improved $P S Q I$ global score $(\mathrm{RCl}<-1.96)$.

Significantly improved

PSQI global score $(\mathrm{RCI}<-\mathbf{1 . 9 6})$

\begin{tabular}{l|l|l|l|}
\hline \multirow{3}{*}{ PSQI ITT } & MAD & CPAP & $P$ \\
\cline { 2 - 4 } & $38.6 \%$ & $16.7 \%$ & .014 \\
& $(17 / 44)$ & $(9 / 54)$ & \\
\cline { 2 - 4 } PSQI PP & $\begin{array}{l}45.7 \% \\
16 / 35)\end{array}$ & $\begin{array}{l}19.0 \% \\
(4 / 21)\end{array}$ & .044 \\
\cline { 2 - 4 } & $(16 / 21)$ & \\
\cline { 2 - 4 } & & &
\end{tabular}

ITT, intention to treat; PP, per protocol; P, Pearson chisquare test; PSQI, Pittsburgh Sleep Quality Index (global score); $\mathrm{RCl}$, reliable change index.

\section{Respiratory Event Index}

Both treatment groups had a reduction in median REI from baseline to follow-up: MAD group from 16.3 (12.4 $23.0)$ to $7.9(6.0-13.7), P<0.001$ and CPAP group from $18.1(15.3-24.6)$ to $1.1(0.6-1.6), P<0.001$ (Table 2$)$. The CPAP group had a lower REI score than the MAD group at follow-up $(P<0.001)$.

\section{DISCUSSION}

In this randomized controlled clinical trial we compared the effect of CPAP and MAD treatment on selfreported sleep quality in patients with mild and moderate OSA. After 4 months of treatment, self-reported sleep quality was improved in both treatment groups. Although PSQI global score at follow-up was similar between the treatment groups, the difference in the number of patients reporting a reliable improvement in PSQI global score was significant. Patients reporting an improved PSQI global score according to the RCI are assumed to also have clinically improved sleep quality from baseline to followup. ${ }^{33}$ This means that $38.6 \%$ of patients using a MAD and $16.7 \%$ of patients using CPAP in our trial experienced an improvement in perceived sleep quality, even though the absolute change in PSQI global score seem modest. By using the RCI, we counteract the effect from possible outliers in PSQI change, thereby limiting the possible influence from factors not related to OSA on the PSQI change at group level.

It is reasonable to believe that effective treatment of OSA should improve sleep quality. ${ }^{24,34}$ Both treatment groups in this trial significantly reduced the REI, indicating that both MAD and CPAP have a considerable positive effect on mild and moderate OSA. In the CPAP group the REI was reduced to 1.1, well below the limit for having OSA. ${ }^{4}$ The MAD group had on average a residual REI at 7.9 , which is unsurprising as the MAD by increasing the volume of the upper airway merely reduce the negative respiratory pressure, not fully eliminate the collapsibility of the upper airways. ${ }^{16}$ Although reducing the REI by more than $50 \%$, the residual REI may be contributing to PSQI global score remaining above 5.0 in the MAD group at 
follow-up. However, CPAP treatment also did not reduce PSQI global score below 5.0, showing that the PSQI global score is dependent on more factors than REI.,23 The limited reduction of PSQI global score in the CPAP group may be due to low CPAP compliance in the ITT analysis. However, the PP analysis showed that when only including patients who used the MAD or CPAP more than 4 hours, more than $50 \%$ of nights, more patients using MAD than using CPAP report improved sleep quality according to the RCI (Table 3). This trend was even more pronounced when using 4 hours and $70 \%$ of nights as the lower limit for compliance ( $P=0.02$, table not shown), indicating that MAD treatment, despite having higher residual REI, is better than CPAP treatment at improving self-reported sleep quality. Previous studies have indicated that MAD is perceived as a less invasive treatment alternative and is preferred over CPAP treatment in most studies where patients have been treated with both appliances. ${ }^{8,15,18}$ This is not only in line with our findings regarding treatment compliance, but also indicates why MAD seems to be better at improving sleep quality, given that CPAP treatment may be uncomfortable and thus impair sleep quality while simultaneously lowering REI. ${ }^{35,36}$

This study deviates from the more common cutoff level for treatment compliance being $>4$ hours, $>70 \%$ of nights ${ }^{21}$ because only 33 MAD patients $(76.7 \%)$ and 17 CPAP patients $(31.5 \%)$ would be regarded as compliant with treatment, further reducing statistical power in the PP analyses. Regardless of compliance cutoff level, treatment compliance in this study was better with MAD than with CPAP, a finding that also was found in a recent metaanalysis. ${ }^{37}$ Furthermore, the poor compliance with CPAP treatment in this trial is in line with another meta-analysis where CPAP compliance became worse with reduced OSA severity. ${ }^{21}$ Fortunately, the risk of serious cardiovascular events and death seems to be small in mild and moderate OSA compared to severe OSA. ${ }^{38-40}$ This finding, and the debated role of respiratory events as a predictor for cardiovascular events and early death, suggests that good compliance with treatment may be more important than optimal reduction of respiratory events, as long as the residual REI is within preferably mild and perhaps moderate OSA. ${ }^{41,42}$ Achieving patient- perceived benefits of OSA treatment such as improved sleep quality should therefore be considered part of the treatment goal, especially in mild and moderate OSA, where factors other than disease severity seem to be important for motivation and compliance to treatment. ${ }^{21,35,43}$ To bypass concerns related to treatment compliance, mandibular advancement surgery may be considered in patients in whom REI is effectively reduced and sleep quality is improved with MAD treatment. Surgical mandibular advancement reduces REI through mechanisms similar to MAD treatment, but the invasive nature of such a surgical procedure makes it more suited for patients having severe OSA and should not be considered in patients where there are high risks of postsurgical malocclusion or poor facial esthetics. $^{12,44}$

The randomization procedure in this trial was successful; thus, any first-night effects in the polygraphic sleep recordings ${ }^{26,45}$ or placebo effects on sleep quality in this initial phase of treatment should be equal in the two treatment groups because of the study design. ${ }^{46}$ However, there was a risk of recruitment bias because the study was unblinded and some patients could be familiar with either MAD or CPAP treatment before agreeing to participate in the trial. To minimize this risk, only patients who had not in any way been treated for OSA previously were invited to the trial. Patients in this trial deviated from the Norwegian general population by having higher average body mass index and worse self-reported general health at baseline. ${ }^{47,48}$

One major limitation of this study is the uncertainty of using self-reported compliance. Objectively measured compliance was available for the CPAP group only, which showed minor discrepancies between self-reported and objectively measured compliance for 6 patients. There are no reasons to believe that patients in the MAD group are less honest than those in the CPAP group when reporting on their use of the appliance. Moreover, very good agreement between subjective and objective measured compliance in MAD patients was found in previous studies. $^{49}$ PP analyses using objectively measured compliance for the CPAP group are presented in the supplementary Table S 2 and supplementary Table S3.

Imputed data were used in the primary analysis as recommended by the CONSORT 2010 statement to avoid compromising the methodologic strengths of the randomization. ${ }^{31}$ Results from analysis only including patients with no imputed data are presented in the supplementary Table S 4 and supplementary Table S 5 . Fisher exact test showed that the 6 patients without any information on compliance were similar to the rest of the patients at baseline (data not shown). Furthermore, the 5 patients who were lost to follow-up in the MAD group were missing at random. The missing patients in this trial are therefore unlikely to create any bias at follow-up. Although sleep data on follow-up was available in fewer patients in the MAD group (77.6\%) than in the CPAP group $(89.1 \%)$, this difference was not statistically significant (chi-square test, $P=0.11$ ) and unlikely to affect the results.

\section{CONCLUSION}

In summary, both MAD and CPAP treatment improve self-reported sleep quality in patients with mild and moderate OSA. When using the CPAP device, the CPAP group achieved a lower REI than the MAD group. Nevertheless, significantly more patients in the MAD group comply with the treatment and report a significant improvement in PSQI global score compared to the CPAP group. Regarding sleep quality, MAD should be considered 
a better treatment option than CPAP in mild and moderate OSA.

\section{ABBREVIATIONS}

AHI - apnea-hypopnea index

CPAP - continuous positive airway pressure

ITT - intention to treat

MAD - mandibular advancement device

OSA - obstructive sleep apnea

$\mathrm{PP}$ - per protocol

PSQI - Pittsburgh Sleep Quality Index

$\mathrm{RCI}$ - reliable change index

REI - respiratory event index

\section{ACKNOWLEDGEMENTS}

We would like to thank all medical and dental personnel involved in the data collection at the University Hospital of Northern Norway, St. Olavs University Hospital, and Aleris Hospital and Medical Centre. We offer special thanks to Sølve Hellem and Kerstin Fischer for initiating and participating in the planning of the study.

\section{REFERENCES}

1. American Academy of Sleep Medicine. International Classification of Sleep Disorders, Third Edition. Darien, IL: American Academy of Sleep Medicine; 2014.

2. Lusic Kalcina L, Valic M, Pecotic R, Pavlinac Dodig I, Dogas Z. Good and poor sleepers among OSA patients: sleep quality and overnight polysomnography findings. Neurol Sci. 2017;38(7):12991306.

3. Pires GN, Bezerra AG, Tufik S, Andersen ML. Effects of acute sleep deprivation on state anxiety levels: a systematic review and metaanalysis. Sleep Med. 2016;24:109-118.

4. American Academy of Sleep Medicine. Sleep-related breathing disorders in adults: recommendations for syndrome definition and measurement techniques in clinical research. The Report of an American Academy of Sleep Medicine Task Force. Sleep. 1999;22(5):667-689.

5. American Academy of Sleep Medicine. AASM Style Guide for Sleep Medicine Terminology. Updated November 2015. Darien, IL: American Academy of Sleep Medicine; 2016.

6. Sullivan CE, Berthon-Jones M, Issa FG, Eves L. 'Reversal of obstructive sleep apnoea by continuous positive airway pressure applied through the nares'. Lancet. 1981;317(8225):862-865.

7. Ramar K, Dort LC, Katz SG, et al. Clinical Practice Guideline for the Treatment of Obstructive Sleep Apnea and Snoring with Oral Appliance Therapy: An Update for 2015. J Clin Sleep Med. 2015;11(7):773-827.

Iftikhar IH, Bittencourt L, Youngstedt SD, et al. Comparative efficacy of CPAP, MADs, exercise-training, and dietary weight loss for sleep apnea: a network meta-analysis. Sleep Med. 2017;30:7-14.

9. Ng SSS, Chan RSM, Woo J, et al. A randomized controlled study to examine the effect of a lifestyle modification program in OSA. Chest. 2015;148(5):1193-1203.

10. Qaseem A, Holty JE, Owens DK, et al. Management of obstructive sleep apnea in adults: A clinical practice guideline from the American College of Physicians. Ann Intern Med. 2013;159(7):471-483.
11.

Moxness MH, Nordgard S. An observational cohort study of the effects of septoplasty with or without inferior turbinate reduction in patients with obstructive sleep apnea. BMC Ear Nose Throat Disord. 2014; $14: 11$

John CR, Gandhi S, Sakharia AR, James TT. Maxillomandibular advancement is a successful treatment for obstructive sleep apnoea: a systematic review and meta-analysis. Int J Oral Maxillofac Surg. 2018:47(12):1561-1571.

13. Doff MH, Hoekema A, Wijkstra PJ, et al. Oral appliance versus continuous positive airway pressure in obstructive sleep apnea syndrome: a 2-year follow-up. Sleep. 2013;36(9):1289-1296.

14. Cammaroto G, Galletti C, Galletti F, Galletti B, Galletti C, GayEscoda C. Mandibular advancement devices vs nasal-continuous positive airway pressure in the treatment of obstructive sleep apnoea. Systematic review and meta-analysis. Medicina Oral Patología Oral y Cirugia Bucal. 2017:0-0.

16. Marklund M. Update on Oral Appliance Therapy for OSA. Curr Sleep Med Rep. 2017;3(3):143-151.

17. El-Solh AA, Homish GG, Ditursi G, et al. A randomized crossover trial evaluating continuous positive airway pressure versus mandibular advancement device on health outcomes in veterans with posttraumatic stress disorder. J Clin Sleep Med. 2017;13(11):13271335.

18. Phillips CL, Grunstein RR, Darendeliler MA, et al. Health outcomes of continuous positive airway pressure versus oral appliance treatment for obstructive sleep apnea: a randomized controlled trial. Am J Respir Crit Care Med. 2013;187(8):879-887.

19. Sutherland K, Vanderveken OM, Tsuda H, et al. Oral appliance treatment for obstructive sleep apnea: an update. J Clin Sleep Med. 2014;10(2):215-227.

20. Giles TL, Lasserson TJ, Smith BH, White J, Wright J, Cates CJ. Continuous positive airways pressure for obstructive sleep apnoea in adults. Cochrane Database Syst Rev. 2006(3):CD001106.

21. Madbouly EM, Nadeem R, Nida M, Molnar J, Aggarwal S, Loomba $R$. The role of severity of obstructive sleep apnea measured by apneahypopnea index in predicting compliance with pressure therapy, a meta-analysis. Am J Ther. 2014;21(4):260-264.

22. Ulander M, Johansson MS, Ewaldh AE, Svanborg E, Brostrom A. Side effects to continuous positive airway pressure treatment for obstructive sleep apnoea: changes over time and association to adherence. Sleep Breath. 2014;18(4):799-807.

23. Wu MN, Lai CL, Liu CK, et al. More severe hypoxemia is associated with better subjective sleep quality in obstructive sleep apnea. BMC Pulm Med. 2015;15:117.

24. Loredo JS, Ancoli-Israel S, Kim EJ, Lim WJ, Dimsdale JE. Effect of continuous positive airway pressure versus supplemental oxygen on sleep quality in obstructive sleep apnea: a placebo-CPAP-controlled study. Sleep. 2006;29(4):564-571.

25. Quan SF, Budhiraja R, Kushida CA. Associations between sleep quality, sleep architecture and sleep disordered breathing and memory after continuous positive airway pressure in patients with obstructive sleep apnea in the Apnea Positive Pressure Long-term Efficacy Study (APPLES). Sleep Sci. 2018;11(4):231-238.

26. Kapur VK, Auckley DH, Chowdhuri S, et al. Clinical Practice Guideline for Diagnostic Testing for Adult Obstructive Sleep Apnea: An American Academy of Sleep Medicine Clinical Practice Guideline. J Clin Sleep Med. 2017;13(3):479-504.

27. Kushida CA, Littner MR, Hirshkowitz M, et al. Practice parameters for the use of continuous and bilevel positive airway pressure devices to treat adult patients with sleep-related breathing disorders. Sleep. 2006;29(3):375-380.

28. Buysse DJ, Reynolds III CF, Monk TH, Berman SR, Kupfer DJ. The Pittsburgh Sleep Quality Index: A new instrument for psychiatric 
practice and research. Psychiatry Res. 1989;28:193-213.

29.

Jacobson NS, Truax P. Clinical significance: a statistical approach to defining meaningful change in psychotherapy research. J Consult Clin Psychol. 1991;59(1):12-19.

30.

Hollis S, Campbell F. What is meant by intention to treat analysis? Survey of published randomised controlled trials. BMJ. 1999;319(7211):670-674.

31. Moher D, Hopewell S, Schulz KF, et al. CONSORT 2010 explanation and elaboration: updated guidelines for reporting parallel group randomised trials. BMJ. 2010;340:c869.

Altman DG. Missing outcomes in randomized trials: addressing the dilemma. Open Med. 2009;3(2):e51-53.

33.

Currie SR, Wilson KG, Curran D. Clinical significance and predictors of treatment response to cognitive-behavior therapy for insomnia secondary to chronic pain. J Behav Med. 2002;25(2):135-153.

34. Kline CE, Crowley EP, Ewing GB, et al. The effect of exercise training on obstructive sleep apnea and sleep quality: a randomized controlled trial. Sleep. 2011;34(12):1631-1640.

Olsen S, Smith S, Oei TP. Adherence to continuous positive airway pressure therapy in obstructive sleep apnoea sufferers: a theoretical approach to treatment adherence and intervention. Clin Psychol Rev. 2008;28(8):1355-1371.

Sawyer AM, Gooneratne NS, Marcus CL, Ofer D, Richards KC, Weaver TE. A systematic review of CPAP adherence across age groups: clinical and empiric insights for developing CPAP adherence interventions. Sleep Med Rev. 2011;15(6):343-356.

Schwartz M, Acosta L, Hung YL, Padilla M, Enciso R. Effects of CPAP and mandibular advancement device treatment in obstructive sleep apnea patients: a systematic review and meta-analysis. Sleep Breath. 2018;22(3):555-568.

Marin JM, Carrizo SJ, Vicente E, Agusti AGN. Long-term cardiovascular outcomes in men with obstructive sleep apnoeahypopnoea with or without treatment with continuous positive airway pressure: an observational study. Lancet. 2005;365(9464):1046-1053.

Yaggi HK, Concato J, Kernan WN, Lichtman JH, Brass LM, Mohsenin V. Obstructive sleep apnea as a risk factor for stroke and death. N Engl J Med. 2005;353(19):2034-2041.

40. Anandam A, Patil M, Akinnusi M, Jaoude P, El-Solh AA. Cardiovascular mortality in obstructive sleep apnoea treated with continuous positive airway pressure or oral appliance: an observational study. Respirology. 2013;18(8):1184-1190.

41. Marshall NS, Wong KK, Liu PY, Cullen SR, Knuiman MW, Grunstein RR. Sleep apnea as an independent risk factor for all-cause mortality: the Busselton Health Study. Sleep. 2008;31(8):1079-1085.

Oldenburg O, Wellmann B, Buchholz A, et al. Nocturnal hypoxaemia is associated with increased mortality in stable heart failure patients. Eur Heart J. 2016;37(21):1695-1703.
43. Jacobsen AR, Eriksen F, Hansen RW, et al. Determinants for adherence to continuous positive airway pressure therapy in obstructive sleep apnea. PLoS One. 2017;12(12):e0189614.

44. Zaghi S, Holty JE, Certal V, et al. Maxillomandibular Advancement for Treatment of Obstructive Sleep Apnea: A Meta-analysis. JAMA Otolaryngol Head Neck Surg. 2016;142(1):58-66.

45. Gouveris H, Selivanova O, Bausmer U, Goepel B, Mann W. Firstnight-effect on polysomnographic respiratory sleep parameters in patients with sleep-disordered breathing and upper airway pathology. Eur Arch Otorhinolaryngol. 2010;267(9):1449-1453.

46. Schulz KF. Randomized controlled trials. Clin Obstet Gynecol. 1998;41(2):245-256.

47. Krokstad S, Ernstsen L, Sund ER, et al. Social and spatial patterns of obesity diffusion over three decades in a Norwegian county population: the HUNT Study. BMC Public Health. 2013;13:973.

48. Statistisk sentralbyrå. Dette er Norge 2018. Tall som forteller. www.ssb.no/norge: Statistisk sentralbyrå; September 3rd, 20182018.

49. Dieltjens M, Braem MJ, Vroegop A, et al. Objectively measured vs self-reported compliance during oral appliance therapy for sleepdisordered breathing. Chest. 2013;144(5):1495-1502.

\section{SUBMISSION \& CORRESPONDENCE INFORMATION}

\section{Submitted for publication August 23, 2019 \\ Submitted in final revised form November 26, 2019 \\ Accepted for publication December 13, 2019}

Address correspondence to: Lars Martin Berg, DDS, Center for Oral Health Services and Research, MidNorway (TkMidt), Professor Brochs gate 2, N-7030 Trondheim, Norway, E-mail: lars.m.berg@ uit.no

\section{DISCLOSURE STATEMENT}

The trial was funded by UiT The Arctic University of Norway, the University Hospital in Northern Norway and St. Olavs University Hospital, Trondheim. The authors have no conflicts of interest to disclose. 


\section{SUPPLEMENTARY TABLES}

Table S 1. Distribution of patient characteristics in intention-to-treat and per protocol analysis

\begin{tabular}{|c|c|c|c|c|}
\hline Baseline variables & $\begin{array}{l}\text { MAD ITT } \\
(n=49)\end{array}$ & $\begin{array}{l}\text { CPAP ITT } \\
(n=55)\end{array}$ & $\begin{array}{l}\text { MAD PP } \\
(n=35)\end{array}$ & $\begin{array}{l}\text { CPAP PP } \\
(n=21)\end{array}$ \\
\hline \multirow{3}{*}{$\begin{array}{l}\text { Age at inclusion } \\
\text { BMI at inclusion } \\
\text { Sex }\end{array}$} & $49.6(9.0)$ & $53.3(10.2)$ & $49.9(9.2)$ & $55.1(10.9)$ \\
\hline & $32.4(7.2)$ & $30.8(6.2)$ & $32.2(7.1)$ & $30.4(5.1)$ \\
\hline & & & & \\
\hline Female & $20(40.8)$ & $17(30.9)$ & $13(37.1)$ & $7(33.3)$ \\
\hline Male & $29(59.2)$ & $38(69.1)$ & $22(62.9)$ & $14(66.7)$ \\
\hline \multicolumn{5}{|l|}{ Marital status } \\
\hline Cohabitant & $37(75.5)$ & $44(80.0)$ & $27(77.1)$ & $19(90.5)$ \\
\hline Living alone & $12(24.5)$ & $11(20.0)$ & $8(22.9)$ & $2(9.5)$ \\
\hline \multicolumn{5}{|l|}{ OSA severity } \\
\hline Mild & $19(38.8)$ & $13(23.6)$ & $15(42.9)$ & $4(19.0)$ \\
\hline Moderate & $30(61.2)$ & $42(76.4)$ & $20(57.1)$ & $17(81.0)$ \\
\hline \multicolumn{5}{|l|}{ Allergy } \\
\hline Yes & $8(16.3)$ & $9(16.4)$ & $3(8.6)$ & $4(19.0)$ \\
\hline No & $51(83.7)$ & $46(83.6)$ & $32(91.4)$ & $17(81.0)$ \\
\hline \multicolumn{5}{|l|}{ Self-reported health } \\
\hline Good-Excellent & $13(26.5)$ & $16(29.1)$ & $13(37.1)$ & $6(28.6)$ \\
\hline Poor-Fair & $36(73.5)$ & $39(70.9)$ & $22(62.9)$ & $15(71.4)$ \\
\hline \multicolumn{5}{|l|}{ Education level } \\
\hline College or university & $27(55.1)$ & $23(41.8)$ & $19(54.3)$ & $8(38.1)$ \\
\hline Other education & $22(44.9)$ & $32(58.2)$ & $16(45.7)$ & $13(61.9)$ \\
\hline \multicolumn{5}{|l|}{ Alcohol consumption } \\
\hline$\leq$ Once per week & $\begin{array}{l}40(81.6) \\
9(184)\end{array}$ & $43(78.2)$ & $\begin{array}{l}29(82.9) \\
6(17.1)\end{array}$ & $\begin{array}{l}17(81.0) \\
4(190)\end{array}$ \\
\hline \multicolumn{5}{|l|}{ Smoking status } \\
\hline Nonsmoking & $42(85.7)$ & $41(74.5)$ & $31(88.6)$ & $19(90.5)$ \\
\hline Smoking & $7(14.3)$ & $14(25.5)$ & $4(11.4)$ & $2(9.5)$ \\
\hline
\end{tabular}

$\mathrm{BMI}$, body mass index $\left(\mathrm{kg} / \mathrm{m}^{2}\right)$; CPAP, continuous positive airway pressure; ITT, intention to treat; MAD, mandibular advancement device; OSA, obstructive sleep apnea; PP, per protocol.

aMean (standard deviation), all other variables: $\mathrm{n}(\%)$.

Table S 2. PSQI and REI at baseline and follow-up (Objectively measured CPAP compliance and self-reported MAD compliance, $>4$ hours, $>50 \%$ of nights).

\begin{tabular}{|c|c|c|c|c|c|}
\hline & \multicolumn{2}{|c|}{ Baseline } & \multicolumn{3}{|c|}{ Follow-up (4 months) } \\
\hline & $\begin{array}{l}\text { MAD } \\
(n=49)\end{array}$ & $\begin{array}{l}\text { CPAP } \\
(n=55)\end{array}$ & $\begin{array}{l}\text { MAD } \\
(n=44)\end{array}$ & $\begin{array}{l}\text { CPAP } \\
(n=54)\end{array}$ & $P$ \\
\hline$P S Q I P P^{b}$ & $\begin{array}{l}8.1 \\
(3.1)\end{array}$ & $\begin{array}{l}6.7 \\
(3.4)\end{array}$ & $\begin{array}{l}5.3^{\mathrm{a}} \\
(2.5)\end{array}$ & $\begin{array}{l}5.5 \\
(3.0)\end{array}$ & .81 \\
\hline
\end{tabular}

CPAP, continuous positive airway pressure; MAD, mandibular advancement device; PP, per protocol; PSQI, Pittsburgh Sleep Quality Index (global score). P indicates t-test between MAD and CPAP group at follow-up.

aStatistically significant change from baseline within treatment group $(P<0.05)$.

bMAD $n=35$ at follow-up, CPAP $n=17$ at follow-up. 
Table S 3. Number of patients with significantly improved PSQI global score $(\mathrm{RCl}<-$ 1.96) (objectively measured CPAP compliance and self-reported MAD compliance, $>4$ hours, $>50 \%$ of nights).

\begin{tabular}{c|l|l|l|}
\multicolumn{4}{|c}{ Improved PSQI global score } \\
\hline \multirow{3}{*}{ PSQI ITT } & MAD & CPAP & $P$ \\
\cline { 2 - 4 } & $38.6 \%$ & $16.7 \%$ & .014 \\
& $(17 / 44)$ & $(9 / 54)$ & \\
PSQI PP & $45.7 \%$ & $17.6 \%$ & .049 \\
& $(16 / 35)$ & $(3 / 17)$ & \\
\cline { 2 - 4 }
\end{tabular}

CPAP, continuous positive airway pressure; ITT, intention to treat; MAD, mandibular advancement device; PP, per protocol; PSQI, Pittsburgh Sleep Quality Index (global score). $P$ indicates Pearson chi-square test.

Table S 4. PSQI and REI at baseline and follow-up (no patients with imputed data).

\begin{tabular}{|c|c|c|c|c|c|}
\hline \multicolumn{3}{|c|}{ Baseline } & \multicolumn{3}{|c|}{ Follow-up (4 months) } \\
\hline & $\begin{array}{l}\text { MAD } \\
(n=43)\end{array}$ & $\begin{array}{l}\text { CPAP } \\
(n=46)\end{array}$ & $\begin{array}{l}\text { MAD } \\
(n=38)\end{array}$ & $\begin{array}{l}\text { CPAP } \\
(n=46)\end{array}$ & $P$ \\
\hline PSQI ITT & $\begin{array}{l}7.7 \\
(2.9)\end{array}$ & $\begin{array}{l}7.8 \\
(3.6)\end{array}$ & $\begin{array}{l}5.4^{a} \\
(2.3)\end{array}$ & $\begin{array}{l}6.9^{a} \\
(3.6)\end{array}$ & .026 \\
\hline$P S Q I P P^{b}$ & $\begin{array}{l}7.8 \\
(2.7)\end{array}$ & $\begin{array}{l}6.7 \\
(3.5)\end{array}$ & $\begin{array}{l}5.2^{\mathrm{a}} \\
(2.3)\end{array}$ & $\begin{array}{l}5.5 \\
(3.1)\end{array}$ & .74 \\
\hline$R E I^{c}$ & $\begin{array}{l}17.1 \\
(12.5-22.8)\end{array}$ & $\begin{array}{l}17.5 \\
(14.6-23.0)\end{array}$ & $\begin{array}{l}7.9^{a} \\
(6.0-13.2)\end{array}$ & $\begin{array}{l}1.1^{a} \\
(0.6-1.6)\end{array}$ & $<.001$ \\
\hline
\end{tabular}

CPAP, continuous positive airway pressure; ITT, intention to treat; MAD, mandibular advancement device; PP, per protocol; PSQI, Pittsburgh Sleep Quality Index (global score); REl, respiratory event index (events/h). $P$ indicates Difference between MAD and CPAP group at follow-up.

aStatistically significant change from baseline within treatment group $(P<0.05)$.

bMAD $n=33$, CPAP $n=16$.

cMAD $n=36$ at follow-up, CPAP $n=43$ at follow-up.

Table S 5. Number of patients with significantly improved PSQI global score $(\mathrm{RCl}<1.96)$ (no patients with imputed data).

Statistically significant

improvement

\begin{tabular}{|l|l|l|l|}
\multirow{4}{*}{ PSQI ITT } & MAD & CPAP & $P$ \\
\cline { 2 - 4 } & $39.5 \%$ & $17.4 \%$ & .024 \\
PSQI PP & $(15 / 38)$ & $(8 / 46)$ & \\
\cline { 2 - 4 } & $42.4 \%$ & $18.8 \%$ & .10 \\
& $(14 / 33)$ & $(3 / 16)$ & \\
\hline
\end{tabular}

CPAP, continuous positive airway pressure; ITT, intention to treat; MAD, mandibular advancement device; PP, per protocol; PSQI, Pittsburgh Sleep Quality Index (global score). $P$ indicates Pearson chi-square test. 